S. Appuni \& H. F. Hamisi. (2013). Is Millennium Development Goal 4 achievable in Tanzania?

Public Health Care, 127: 593-596.

http://dx.doi.org/10.1016/i.puhe.2013.01.016

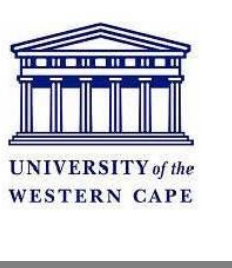

\title{
Is Millennium Development Goal 4 achievable in Tanzania?
}

Sathiya Susuman Appunni and Hamisi F. Hamisi

Child mortality is a fundamental indicator of the level of child health and maternal health. Investigating their health and well-being is an investment for development of any nation. However, approximately 10 million children die each year, mainly from preventable and curable diseases [e.g. pneumonia, diarrhoea, malaria, measles, human immunodeficiency virus/acquired immunodeficiency syndrome (HIV/AIDS)] with low-cost technologies. More than half of these deaths occur in poor and less-developed countries such as Tanzania. Reducing the rate of child mortality has been a challenging issue for most of the less-developed countries, especially in Sub-Saharan Africa. Many strategies and policies have been put into place to further reduce the burden of child mortality, but the most pronounced are Millennium Development Goals (MDGs) 4, 5 and 6, which deal with health issues.

In general, MDGs are the blueprints adopted for a better world, and they pledge to spare no effort in fulfilling that vision. There are eight goals to be achieved by 2015: eradicating extreme poverty; achieving universal primary education; promoting gender equality and the empowerment of women; reducing child mortality; improving maternal health; combatting HIV/AIDS, malaria and other diseases; ensuring environmental sustainability; and developing a global partnership for development. These goals were drawn from the action and targets contained in the Millennium Declaration, which was adopted in September 2000 by 189 nations and signed by 147 heads of state and government during the United Nations Millennium Summit. Behind the MDGs are some specific, measurable, time-bound target (i.e. until 2015) characteristics that bind countries to doing more to fight inadequate income; widespread hunger; gender inequality; environmental deterioration; and lack of education, health care and clean water.

This paper will focus on MDG4, which is concerned with reducing child mortality. Childhood mortality rates are the leading indicator of the level of child health and overall development of a country; and MDG4 aims to reduce the childhood mortality rate by two-thirds by 2015. The target is to reduce the number of children dying before 5 years of age from 93 per 1000 in 1990 to 31 per 1000 in 2015. Thus, MDG4 aims to reduce under-five mortality rates and 
infant mortality rates, which could be achieved by increasing the proportion of 12month-old children who are immunized against measles, BCG, DPT, etc.

Like other developing countries in Sub-Saharan Africa, Tanzania's under-five mortality rate is higher than the world average. In 2009, the under-five mortality rate in Tanzania ranked 27th in terms of severity, at 108 per 1000 live births. There are many and varied factors responsible for the high under-five mortality rate in Tanzania. To fully understand how these factors operate it would be necessary to apply the Mosley-Chen (1984) framework for studying child survival in developing countries. 4 However, this is not within the scope of this short article. In brief, a high burden of diseases such as malaria and HIV/AIDS are the contributing factors for the high under-five mortality rate in the country. For many years, malaria has been the leading cause of death among the population, particularly among children under 5 years of age. For example, in 2009, the National Bureau of Statistics reported that approximately $44 \%$ of childhood mortality was attributed to malaria. However, surveys and other research have indicated a decline in childhood mortality in Tanzania. This study investigated whether the reduction in the childhood mortality rate is sufficiently rapid to achieve the MDG4 target in Tanzania.

Journal articles, reports and related literature by the Tanzania Demographic and Health Survey, United Nations, United Nations Development Programme, World Health Organization, National Bureau of Statistics, Millennium Development Goals Report, United Nations Children Fund, etc. were used as secondary information. Indicators to measure the achievement of MDG4 were made available for areas where childhood mortality is reduced to acceptable levels.

TDHSs show that the under-five mortality rate has decreased significantly from 141 per 1000 live births in 1992 to 81 per 1000 live births in 2010 (see Fig. 1). Similarly, another study showed that the infant mortality rate ranged between 74 per 1000 live births and 131 per 1000 live births for 1994e1999, and dropped to approximately 65 per 1000 live births in the best regions and 116 per 1000 live births in the worst regions between 1999 and 2002. The study indicated that the under-five mortality rate declined from 103 per 1000 live births in 1994 e1999 to 88 per 1000 live births in 1999e2002. Further results showed that under-five mortality in the worst regions dropped from 209 per 1000 live births to 185 per 1000 live births in the same period. The target for the under-five mortality rate in Tanzania by $2015^{5}$ is set at 48 per 1000 live births.

The authors used the data from all previous TDHSs and extracted a trend line to get the linear equation $\mathrm{f}(\mathrm{x}) 1 / 4-13.51 \mathrm{x} \mathrm{p} 165.47$ in order to ascertain if Tanzania could achieve its MDG4 target by 2015. Using this equation, it appears that the under-five mortality rate in 2015 will be 71 per 1000 live births. This is not consistent with the expected target rate of 48 per 1000 live births.

It should, however, be noted that different pictures can be highlighted when focussing on two of Tanzania's regions, such as Mainland Tanzania and 
Zanzibar, and evaluating their progress towards achievement of the MDG4 target. Mainland Tanzania had a child mortality rate of approximately 191 per 1000 live births in 1990; that number was reduced to approximately 112 per 1000 live births in 2008, while the target was 99.6 per 1000 (the reduction was less than expected). This rate is expected to decline further to a target of 64 per 1000 live births by 2015. In contrast, Zanzibar had a child mortality rate of approximately 202 per 1000 live births in 1990; that number was reduced substantially to approximately 101 per 1000 live births in 2008 (better than the expected 105 per 1000 live births). Between 1990 and 2008, Zanzibar reduced its child mortality rate by approximately 50\%, while Mainland Tanzania reduced its child mortality rate by only $41 \%$. Further studies indicate infant mortality rates declining from 115 per 1000 live births in 1990 to 99 per 1000 live births in 2000, and 68 per 1000 live births in 2008 for Mainland Tanzania, which is, again, less than the target of 59 per 1000 live births. In Zanzibar, the rate declined from 120 per 1000 live births in 1990 to 89 per 1000 live births in 2000, and 61 per 1000 live births in 2008, which was better than the target of 62.4 per 1000 live births. One should, however, bear in mind that child survival has increased in Tanzania since the MDG4 target was set. Although this may not be of significance statistically, it may help the target become achievable by 2015 . Selected factors that have contributed to the increased child survival in Tanzania include:

- interventions to reduce malaria (for children and pregnant mothers): ownership of any type of sleeping net increased from $46 \%$ per household in 2004-2005 to approximately $75 \%$ in $2009-2010$;

- immunization: vaccination coverage of children aged 12-23 months increased from $71 \%$ in $2004-2005$ to $75 \%$ in 2010 , and approximately $75 \%$ of children aged $12-$ 23 months are fully vaccinated; and

- vitamin A supplementation: nearly $60 \%$ of 10 children aged 6e59 months received vitamin A supplementation for the 6 months preceding TDHS 2009e2010.

The measurement indicators used to assess the achievability of childhood mortality include the under-five mortality rate, the infant mortality rate and the proportion of 1-year-old children immunized against measles, BCG, DPT, etc. The general trend and status of these indicators are in some ways impressive; the under-five mortality rate and infant mortality rate have been reduced substantially, while immunization coverage against measles has increased. 


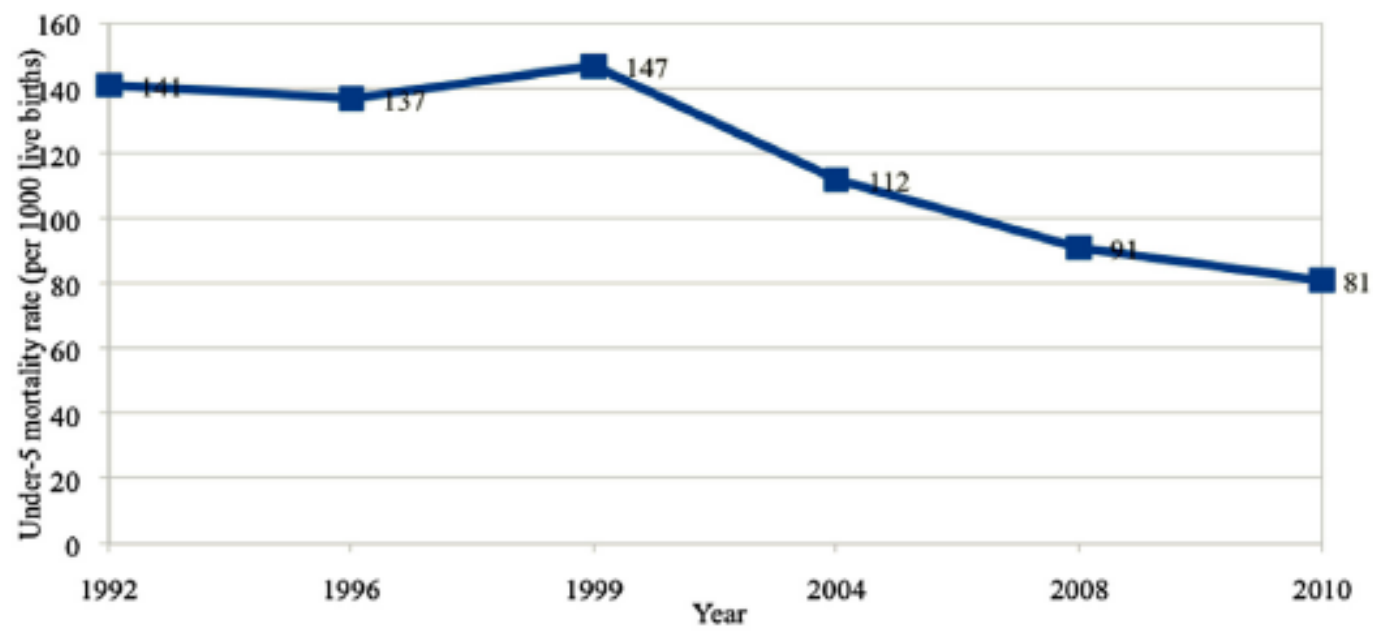

Fig. 1 e National progress towards achievement of Millennium Development Goal 4 in Tanzania. Source: Tanzania Demographic and Health Surveys (1992e2010).

However, it has been shown that the mortality rate in Tanzania declined at a rate of less than $4 \%$ per annum. If Tanzania could raise its annual reduction rate to approximately $5 \%$ per annum, it could possibly meet the MDG4 target by 2015. Contrary to this view, the DG report (2007) highlighted that an average annual reduction rate of approximately 7.8\% would be required for Tanzania to achieve its MDG4 target. The current rate of reduction is insufficient to achieve the MDG4 target by 2015, and Tanzania is among the 60 high-priority developing countries that are not on track to meet the MDG4 target. Using the results from the TDHS 1992 survey, the under-five mortality rate in Tanzania reduced from 141 per 1000 live births in 1992 to 81 per 1000 in 2010; it is unlikely that this number will decrease sharply to 48 per 1000 by 2015 . However, using the child mortality rates of each TDHS, Tanzania was supposed to reduce the mortality rate in 1992 by $40 \%$ to achieve 83 per 1000 live births in 2004e2005. This rate needed to be further reduced by approximately $58 \%$ to reach a rate of 59 per 1000 live births in 2007e2008. From 2007 to 2008 , it could be easily reduced to 48 per 1000 live births in 2015.

However, vital statistics on child mortality in developing countries, especially SubSaharan Africa, are lacking, and data, if available, are far from perfect. As such, demographers, statisticians and health researchers are more likely to rely on indirect estimates using data from surveillance sites or longitudinal records to ascertain the trends in child mortality. Careful attention must be paid to these matters when the data are analyzed and reported, as the data can reveal different interpretations depending on the methodology used. DHS managed to unload some considerations to be taken when TDHSs are used to estimate the achievability of the MDGs in Tanzania, including MDG4.

MDG4 is still achievable in Tanzania, but the critical issue is how to transform the pace of change from what has been seen over the last decade into dramatically faster progress. Tanzania has managed to reduce its under-five mortality rate from 141 per 1000 live births in 1992 to 81 per 1000 live births in 2010, and the proportion of children immunized against measles has increased from $71 \%$ of children aged $12 \mathrm{e} 23$ months in 2004e2005 to $75 \%$ in 2010. Unfortunately, there is regional variation in the rate of decline in underfive mortality in Tanzania. For example, Zanzibar shows substantial reduction and is on 
track to achieve MDG4 if one consider achievements at the regional level. In contrast, Mainland Tanzania is not on track to achieve its target as a region. There are some issues that delay most of the developing countries in general. Tanzania is particular in reducing its child mortality burden. Underfunding in the health system is one of the greatest challenges in Tanzania; for example, public expenditure on health is $2.4 \%$ of the gross national product or US $\$ 7.6$ per person. This creates problems in infrastructure, procurement of equipment, and availability of drugs in health centres. Also, substantial spatial differences in socio- economic status between rural and urban areas, and between regions pose other challenges. For example, children are more likely to die or become malnourished before reaching their fifth birthday in poor areas and in poor families. The use of delivery services varies widely from $32 \%$ in rural areas to $90 \%$ in Dar es Salaam region.

It could be argued that plenty of external donors are helping by donating funds and other resources to ensure that the under-five mortality rate is reduced substantially in Tanzania. Through this financial support, malaria has become considerably more controlled, and improvements have been seen in the fight against measles and other childhood infections. One should keep in mind that most of the gains seen through external donors may be short-lived if funding gaps are not bridged and are conditional. Furthermore, these sources of aid aim to treat the effect and not the root causes of child mortality. To this end, there is a need to determine why children get diseases and why they are malnourished. Last but not least, the literature has shown that more than half of the under-five mortality rate is constituted of infant deaths, and in turn, half of the infant mortality rate is constituted of neonatal deaths. Therefore, the promotion of institutional delivery, and increasing and improving antenatal and post- partum care might reduce the infant mortality rate and, in turn, the under-five mortality rate. It is imperative to promote institutional delivery in rural areas.

Tanzania may still be considered to be on track to achieve the MDG4 target by 2015 if the above-mentioned issues are taken into consideration.

\section{Acknowledgements}

The authors would like to thank various data providers, especially the TDHS and the University of the Western Cape, Cape Town, South Africa. 


\section{References}

1. Handa S, Koch S, Wen Ng S. Child mortality in Eastern and Southern Africa. J Popul Rev 2010;49.

2. United Nations. Millennium Development Goals series of publication report. Available at: www.un.org; 2009.

3. UNICEF. Millennium Development Goals: reduce child mortality. Available at: http://www.unicef.org/mdg/childmortality.html; 2008.

4. Mosley WH, Chen LC. An analytical framework for the study of child survival in developing countries. Popul Dev Rev 1984;10:24e45.

5. Abdulla S, Kessy F. Progress and challenges in reducing maternal and child mortality in Tanzania. Dar es Salaam: Ifakara Health Institute; 2010.

6. Masanja H, De Savigny D, Smithson P, Schellenberg J, John T, Mbuya C. Child survival gains in Tanzania: analysis of data from demographic and health surveys. Lancet 2008;371:1276e83.

7. Tanzania Demographic and Health Survey. Available at: http://www.measuredhs.com; 2010.

8. Janson A. Shed some light on darkness: will Tanzania reach the Millennium Development Goals? Acta Paediatr 2007;96:781e6.

9. World Health Organization. Millennium Development Goal 4: reduce child mortality. Available at: http://www.who.int/topics/millennium_development_goals; 2006.

10. Kabudula CW. The impact of HIV/AIDS on under-five mortality in Malawi. Unpublished Master of Science Dissertation. University of the Western Cape; 2007. 